\title{
A game of Whack-A-Mole: closing multiple ventricular septal defects
}

\author{
Sandeep Sainathan ${ }^{1}$ and Leonardo Mulinari ${ }^{1}$ \\ ${ }^{1}$ University of Miami Miller School of Medicine
}

November 19, 2021

\begin{abstract}
Multiple ventricular septal defects (m-VSD), are a challenging clinical problem. m-VSD can be onerous to manage. Besides the inability to close all the defects in one operative setting due to inadequate visualization, previously undetected defects may become clinically apparent after the closure of the dominant defects, leading to inadequate ventricular septation. This increases the morbidity from the progression of pulmonary hypertension, persistence of congestive cardiac failure, higher incidence of postoperative heart block, and the need for reoperations.
\end{abstract}

A game of Whack-A-Mole: closing multiple ventricular septal defects

By their expert review of the existing literature on multiple ventricular septal defects (m-VSD), Chowdhury and colleagues [1] have described a systematic approach to this challenging clinical problem. m-VSD can be onerous to manage. Besides the inability to close all the defects in one operative setting due to inadequate visualization, previously undetected defects may become clinically apparent after the closure of the dominant defects, leading to inadequate ventricular septation. This increases the morbidity from the progression of pulmonary hypertension, persistence of congestive cardiac failure, higher incidence of postoperative heart block, and the need for reoperations. As alluded to in their manuscript, this can translate to an overall increase in operative mortality compared to an isolated perimembranous VSD.

The authors have nicely divided m-VSD into the following morphological types: multiple discrete muscular defects, combined perimembranous and inlet muscular septal defect, apical m-VSD, spurious m-VSD, and Swiss-cheese defects. Classifying the defects into such morphological types has a clinical bearing on these defects' approach and closure techniques.

While it is usual to start with conventional echocardiography to characterize these defects, further valuable information can be obtained with novel echocardiographic methods such as an "en-face" imaging of the ventricular septum. Cross-sectional imaging with CT angiography can help further delineate the defects and help with operative planning. Similarly, 3-D printing can also add valuable information in some instances $[2]$.

The majority of these defects can be approached via a trans-tricuspid approach, but certain defects such as apical m-VSD may require a ventriculotomy [3]. Surgical techniques can involve the closure of each defect to simultaneous coverage of multiple defects in proximity with a large patch. At times, the defects may need to be obliterated en-masse, especially when they are centered in the apical septum. Again, understanding the morphology of the m-VSD can have critical implications on surgical approaches and outcomes. The spurious m-VSD type, which has multiple openings on the right ventricular side, has a single opening on the left ventricular side and can be elegantly closed from that side. In the combined perimembranous and inlet muscular septal defect type, the conduction pathway is located between these defects and is in jeopardy if incorporated in the repair. 
Options also exist for using a septal occluder device in conjunction with operative closure as a hybrid strategy. Such a technique may help with the increased overall success of the procedure by facilitating closure of difficult-to-reach defects by an open method, avoiding additional incisions such as a ventriculotomy, thus preserving ventricular function and helping decrease operative times. However, septal occluder device should be used with caution, particularly in small hearts, and when more than one occluder device is required, as it can induce acute diastolic dysfunction by decreasing the ventricular cavity size from overcrowding or by tethering the ventricular free wall $[4,5]$.

While the optimal goal of ventricular septation would be no residual shunting and preservation of both the systolic and diastolic ventricular function, this may not always be attainable. Thus, an alternative goal would be to protect the pulmonary vascular bed by pulmonary artery banding. Apart from protecting the pulmonary vascular bed, PA banding facilitates closure of some of the defects spontaneously, may help with better access to the defects in the future by allowing for somatic growth of small hearts, help abbreviate the operative procedure in cases where there are complex associated cardiac defects, and help bail out when all hemodynamically significant defects cannot be closed in one operative setting. Finally, if the ventricular septum is not deemed septatable, such as in a Swiss cheese type [3], protecting the pulmonary vascular bed may allow pursuing a single ventricle pathway.

When possible, primary definitive repair should be the goal, as it is associated with better outcomes than PA banding with or without delayed closure of the m-VSD defects $[3,6]$. Unfortunately, some of the m-VSD patients have or acquire severe ventricular dysfunction after repair and may need cardiac transplantation.

1. Chowdhury U, Anderson R, Spicer D E et al. A review of the therapeutic management of multiple ventricular septal defects. J Card Surg; in press

2. Milano EG, Capelli C, Wray J, Biffi B, Layton S, Lee M, Caputo M, Taylor AM, Schievano S, Biglino G. Current and future applications of 3D printing in congenital cardiology and cardiac surgery. Br $\mathrm{J}$ Radiol. 2019 Feb;92(1094):20180389.

3. Seddio F, Reddy VM, McElhinney DB, Tworetzky W, Silverman NH, Hanley FL. Multiple ventricular septal defects: how and when should they be repaired? J Thorac Cardiovasc Surg. 1999 Jan;117(1):1349

4. Pedra CA, Pedra SR, Chaccur P, Jatene M, Costa RN, Hijazi ZM, Amin Z. Periventricular device closure of congenital muscular ventricular septal defects. Expert Rev Cardiovasc Ther. 2010 May;8(5):66374 .

5. Kumar TK, Knott-Craig CJ. Rare life-threatening complication of device closure of ventricular septal defect in a child. J Thorac Cardiovasc Surg. 2016 May;151(5):e85-6.

6. Kitagawa T, Durham LA 3rd, Mosca RS, Bove EL. Techniques and results in the management of multiple ventricular septal defects. J Thorac Cardiovasc Surg. 1998 Apr;115(4):848-56. 